\title{
Platform Design of Engineering Innovation Experiment Based on WebGIS
}

\author{
Qingjie Zhu ${ }^{1, a,{ }^{*}}$, Xue $\mathrm{Li}^{1, \mathrm{~b}}$ and Bingsheng Zhang ${ }^{1, \mathrm{c}}$ \\ ${ }^{1}$ School of Petroleum Engineering, Changzhou University, Changzhou, Jiangsu Province, China

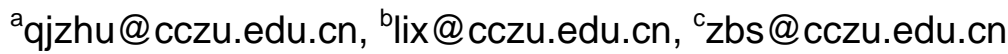 \\ ${ }^{*}$ Corresponding author
}

\begin{abstract}
With the progress of internet and distance interactive teaching, it is urgently necessary to investigate the internet sharing design for open innovation experiment in engineering education. Through platform designing of innovation experiment based on Web GIS, the complex professional functions in the browser and the multiple interactions between the browser/server for engineering innovation experiment are fulfilled. According to the requirements of engineering innovation experiment, internet sharing scheme and browser/server platform structure with professional function are designed. Application programs are developed with JavaScript and VBScript as Active Server Page (ASP). As an application example, the technology function and network interaction ability of the browser application are introduced, and these features can compare favorably with professional software. Finally, the application results are analyzed and some advice is proposed.
\end{abstract}

Keywords: Innovation experiment, WebGIS, Internet sharing, Designing, Engineering education.

\author{
基于WebGIS的工程创新实验设计

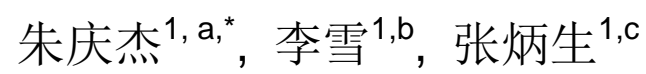 \\ 1常州大学石油工程学院, 常州, 江苏省, 中国 \\ aqjzhu@cczu.edu.cn, blix@cczu.edu.cn, ${ }^{\mathrm{c}} z \mathrm{~b} s @ c c z u . e d u . c n$ \\ “通讯作者
}

中文摘要. 随着互联网技术的进步和远程 互动教育的发展, 对工程创新开放实验的网 络互动设计提出了迫切需要。通过基于 WebGIS的创新实验平台设计, 可以充分满 足创新实验对浏览器端复杂专业功能的要 求, 并实现汶览器/服务器的多元互动。依 据工程创新实验的特点, 设计了包括专业服 务器的汶览器/服务器创新实验平台结构和
网络共享方案。在ASP环境下使用JavaScript 和VBScript语言开发了终端应用程序, 结合 具体实例, 介绍了终端应用的强大技术功能 和网络互动能力, 这样通过浏览器端就可以 实现空间分析和综合评价等复杂的专业计 算功能。最后分析了创新实验平台的应用效 果并对实验平台开发提出几点建议。 
关键词: 创新实验; WebGIS; 网络共享; 设 计; 工程教育

\section{1. 引言}

伴随着教育信息化发展, 开放的工程创 新实验需要研究网络共享和互动的技术与 方法。在网络共享技术中, 近年来基于Web 的教育和知识共享环境构建发展迅速, 是对 实验个人环境设计的前所未有的挑战 ${ }^{[1-3]}$ 。 现在, 通过互联网人们可以享受交通查询、 地图显示、信息查询等各种各样的服务。但 创新工程实验与一般的网络信息共享有着 很大区别, 其一是工程创新实验需要在汶览 器 (客户端) 实现一般只有专业软件而非常 规网页所能实现的复杂功能; 其二是对网络 互动的需要, 即实现汶览器/服务器互动而 非简单的网络浏览。因此, WebGIS已经成 为工程创新实验网络互动平台设计的必要 技术 ${ }^{[4-7]}$ 。WebGIS, 比如MapXtreme, 可以 将网络和GIS专业分析融合, 进而进行各种 的创新实验分析工作。WebGIS已经在诸多 领域应用, 它在创新实验设计中的进一步应 用, 不仅会改变工程创新实验设计方法, 而 且会改变工程教育的教学方式与方法。

现今的教育信息化研究, 主要集中在基 于Web的远程教学平台设计, 对WebGIS技 术的应用研究相对欠缺。与一般地理信息系 统相比, WebGIS 可以使原来只有局域网或 单机版GIS 软件才能实现的分析功能扩展 到整个互联网区域。通过合理设计, WebGIS 不仅可以实现地理属性数据在全球范围内 的共享, 而且可以实现汶览器端对地理属性 数据的复杂操作, 以及汶览器 (用户) 与服 务器之间的对图形和属性数据的共同分享, 进而实现复杂功能的互动 ${ }^{[8-10]}$ 。

WebGIS指一个在互联网平台运行的地 理信息系统, 客户仅仅采用超文本传输协议 就可以在万维网浏览器上实现空间分析的 地理信息系统, 因此, 也被人们称为互联网 GIS。其通过把HTTP和 TCP/IP等标准引入 到GIS软件, 从而把GIS空间分析的专业技 能得以在汶览器中实现。WebGIS的实现技 术包括CGI, Plug-in, ActiveX, Java, ASP

(ActiveX Server Page) 等。其中用作Web 服务器动态站点设计的ASP技术, 得益于其
互动操作优势而应用最为广泛。ASP是微软 公司开发的Web服务器的动态站点设计技 术。ASP技术很容易实现客户端与服务器端 的动态高效交互, 它可以在各种浏览器运 行, 并可以与HTML紧密集成, 支持ActiveX Server组件。

WebGIS 可以方便的实现数据实时更 新, 以及不同级别与层次的数据管理, 从而 使位于不同地理位置的计算机 (服务器) 可 以协同工作, 为工程创新实验的多地协同特 点提供了方便而又高效的技术解决方案。而 且, 在 $\mathrm{B} / \mathrm{S}$ (Browser/Server, 汶览器/服务器 模）模式下，只需要应用WebGIS做好服务 器端的应用开发, 不需要用户在客户端进行 太多的设计, 就可以实现工程创新实验的互 动设计。这也符合工程创新实验设计所需要 的瘦客户端大服务器的互动教学理念 ${ }^{[11]}$ 。

本文讨论了基于WebGIS 的创新实验平 台设计, 介绍了系统结构、应用模型和终端 程序开发框图。结合具体实例, 在ASP环境 下使用JavaScript和VBScript 语言开发了终 端应用程序, 为工程创新实验的网络开放互 动设计和本科/硕士生综合创新能力的提高 进行了有益尝试, 并为工程创新试验设计方 案研究与远程互动教学提供借鉴。

\section{2. 平台结构设计}

平台设计基于Browser/Server(浏览器/ 服务器) 结构, 简称 $\mathrm{B} / \mathrm{S}$ 结构。 $\mathrm{B} / \mathrm{S}$ 结构是随 着互联网技术的发展, 由C/S (Client/Server, 客户端/服务器）结构的技术改进而来。此 结构模式, 让用户完全通过万维网浏览器来 实现各种操作, 增加了用户端的适应性, 可 以适用于不同的操作系统和浏览器; 包括逻 辑判断和数据分析在内的诸多功能都在服 务器端进行。 $B / S$ 模式具有全球范围内可以 借助互联网随时访问的巨大优势, 且汶览器 端的技术维护也相对简单, 数据共享性也极 其便宜, 当服务器端修改网页时, 所有万维 网用户的网页也将同时更新。B/S结构与 VB Script、JavaScript等多种语言和ActiveX技术 的完美结合, 实现了通过浏览器进行专用软 件的诸多分析功能。

在服务器端采用分层设计, 第一层为 Web层, 负责对用户请求做出相应, 一般使 
用较少的代码以方便页面显示。即该层只负 责接受用户请求并将其向后面各层传递。第 二层逻辑层, 对用户请求做出逻辑判断, 比 如用户操作的合法性和有效性, 通过这一层 的逻辑判断, 决定是否可以进入下一层。第 三层是数据访问层, 这一层封装所有与数据 库相关的代码, 从专业服务器 (或者软件) 传进来的数据在这里封存, 并对合法用户开 放, 使用户得到请求所需的各类数据。

平台下一层为GIS服务器, 进行各类专 业数据分析和专业的实验评价结果。比如 GIS空间决策支持分析、平面分析、以及遥 感图像谱段分析增强等功能。本次设计的工 程创新实验平台结构见图1。

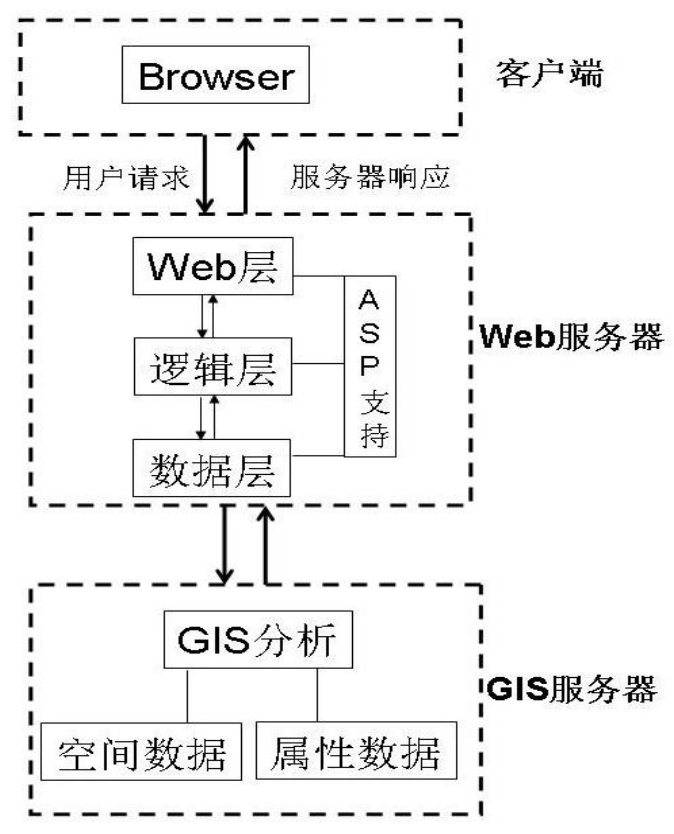

图1 平台体系结构

这样，通过ASP环境的应用程序开发， 使用户通过互联网汶览器发出请求, 并通过 Web 服务器得到GIS服务器的专业分析结 果。并且, 实验中的学生就是网络上的浏览 器客户, 他们通过Web服务器与专业老师、 实验分析软件、实际工程数据等有机结合起 来 (服务器端), 这就相当于Web技术在工 程教育的创新实验实际操作中为实验室和 学生起到桥梁和纽带作用。

\section{3. 应用实例分析}

以一个应用多准则评价方法进行工程 安全评价的实验实例来说明。学生需要根据 给定的影响因素图件 (图层), 通过层次分
析法 (AHP) 来确定权重系数, 在权重系数 计算合理的情况下, 通过多准则评价, 得到 工程的安全评价结果, 见图2。

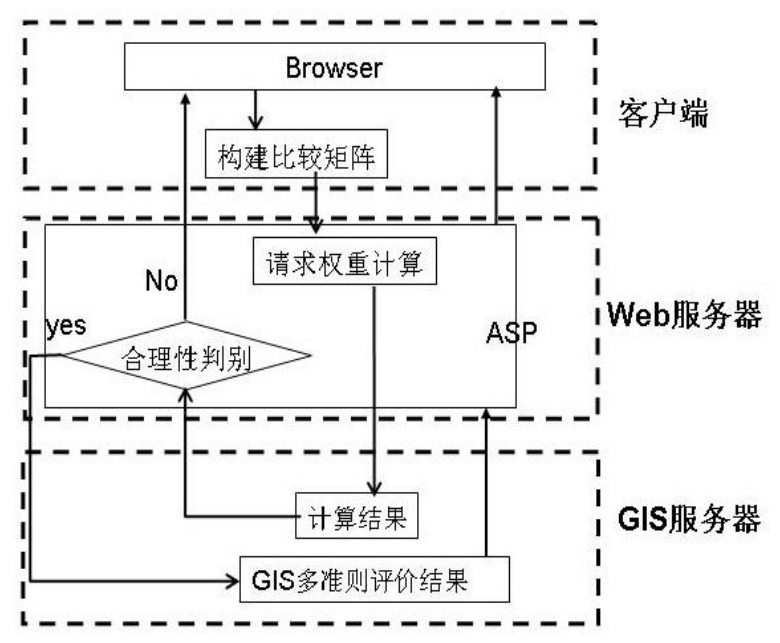

图2 基于Web的实验流程

实验的关键有两个, 一个是学生构建的 比较矩阵通过Web服务器请求GIS服务器进 行权重计算, 并在Web端做出合理性判断, 若不合理则返回学生; 另一个是权重计算合 理的情况下请求GIS 服务器进一步进行多 准则安全评价, 并将评价结果借助Web服务 器返回给实验的学生。权重计算的合理性可 以通过以下比较矩阵的构建过程来做分析。

如表1所示, 以最简单的三个因素的情 况来分析, 比较矩阵的构建过程, 即是影响 因素两两比较重要性的过程。同一因素的重 要性自然相等, 因素1是因素2的重要性的 2 倍, 所以第1行第2列填2, 因素1的重要性是 因素3的6倍, 所以第1行第3列填入6。同样 可以得到其它所有位置的数值。

表1 比较矩阵1构建

\begin{tabular}{|c|c|c|c|}
\hline Factor & Factor1 & Factor2 & Factor3 \\
\hline Factor1 & 1 & 2 & 6 \\
\hline Factor2 & $1 / 2$ & 1 & 4 \\
\hline Factor3 & $1 / 6$ & $1 / 4$ & 1 \\
\hline
\end{tabular}

这样得到的权重系数计算结果是 0.588 , $0.322,0.090$ 。即因素1最重要所以权重最大, 因素3最不重要，所以权重最小。其一致性 比率是 0.0086 。这是一个有不一致但满足精 度需要的结果, 其产生的原因就在于各个因 素之间的比率关系。比如, 从表1中可以看 
出, $F 1 / F 2=2, F 1 / F 3=6$, 那么 $F 2 / F 3=3$ 是可 以推导出来的, 但事实上, 在表 1 中, F2/F3 选择的是 4 , 而不是 3 , 因此, 产生不一致。

这种不一致可以通过构建协调的比较 矩阵来消除, 如我们改变一下表 1 ,如表 2 。

表2 比较矩阵1构建

\begin{tabular}{|c|c|c|c|}
\hline Factor & Factor1 & Factor2 & Factor3 \\
\hline Factor1 & 1 & 2 & 6 \\
\hline Factor2 & $1 / 2$ & 1 & 3 \\
\hline Factor3 & $1 / 6$ & $1 / 3$ & 1 \\
\hline
\end{tabular}

从表2中可以看出, $F 1 / F 2=2, F 1 / F 3=6$, , $F 2 / F 3=3$ 。这时的矩阵是一个完全一致阵, 计算的一致性比率结果为 0 , 即没有出现不 同因素之间的不协调现象。

由此可以看出, 在实验设计中, 必须首 先验证权重系数的结果是否满足一致性要 求。这也正是工程实验与一般网络教学的不 同之处, 工程实验必须满足专业计算的需 要, 实验设计不但需要Web服务器, 还需要 专业 (比如GIS) 服务器; 而且工程创新实 验平台的互动性远大于一般的网络互动, 这 需要在设计中对互动性进行专门考虑, 因 此, 我们在ASP环境开发终端应用程序, 以 实现客户端与服务器端的动态高效互动。

\section{4. 终端应用程序开发}

ASP作为服务器端脚本编写环境, 被广 泛用于交互式应用程序开发。通过ASP, 很 容易做到HTML、脚本命令和COM组件的 融合, 从而创建具有强大功能的交互式Web 应用程序。开发过程见图3。

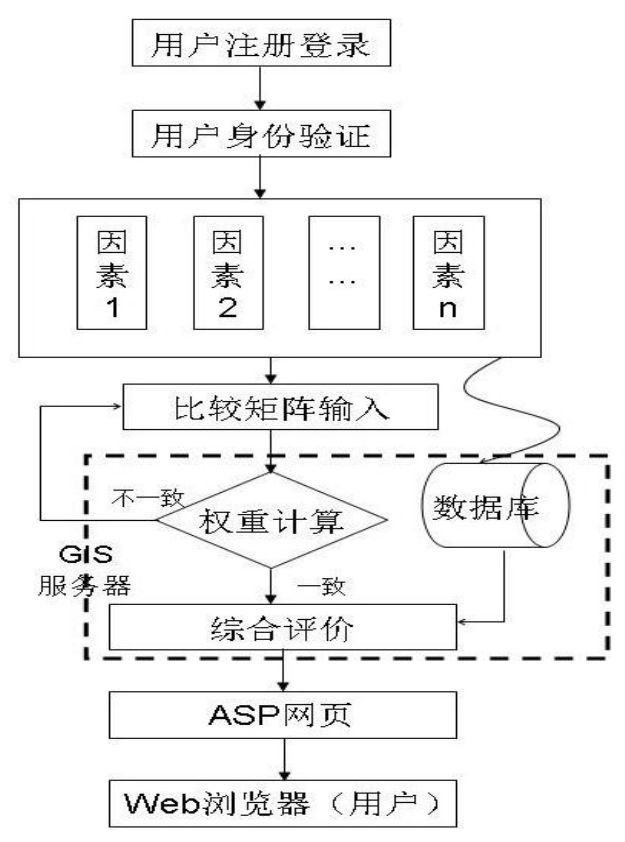

图3应用程序开发框图

应用程序采用模块式结构, 将各应用程 序放在服务组名目录下, 使各模块之间互不 影响, 并可将各模块共用程序放置于一个共 用目录, 以方便各个模块调用。以 “评价结 果” 模块为例, 其目录名为cdpjjg, 实现该 结果访问的应用程序, 除了共用程序外, 还 包括以下程序：（1）获取服务组名、初始 化结果图件引擎的cdpjjg.asp文件; (2) 定 义应用程序中等常量的 cdpjjgConstants.asp 文件;（3）调用Container.asp以用来显示结 果图件的cdpjjgPage.asp文件; （4）工具调 用的Container.asp文件; 以及超链接文件等。 当用户访问评价结果模块时, 系统在经过验 证和逻辑判断后, 访问专业软件获取评价结 果并返回给客户汶览器。如CDpjjgConstants. asp文件代码如下:

<SCRIPT LANGUAGE="VBScript"
RUNAT="Server">
Const cCdServerGroupName = "cdpjjg"
Const cCdBD = "Border"
Const cCdWE = "Well"
Const cCdCDpjjg = "resultspj"
Const cCdPreviousMapState $=$
"PreviousMapState"
Const cCdDefaultCenterX $=0$
Const cCdDefaultCenterY $=0$
Const cCdDefaultZoom $=500$
</SCRIPT>




\section{5. 结束语}

本文针对开放工程创新试验网络交互 性强、客户端功能复杂、需要专业服务器参 与又要远程访问的矛盾特性, 通过基于 Browser/Server结构的平台设计, 避免了局 域网难以远程访问和客户端程序复杂的局 限; 同时, 通过在ASP环境下应用JavaScript 和VBScript语言开发终端应用程序, 克服了 一般网页功能简单、不能进行专业分析的局 限; 并通过将专业服务器与 Web 服务器结 合, 使学生在很容易在个人计算机上借助专 业软件进行复杂的工程实验分析, 并得到专 业软件的计算结果。

但本次工程创新试验网络平台开发尝 试, 仅限于针对具有地理属性的工程问题所 开发的创新试验, 也就是说, 专业服务器软 件是GIS分析软件, 对于需要进一步二次开 发或者更为复杂的专业实验问题, 尚需要进 一步研究。也就是说, 尽管通过创新实验平 台开发尝试找到了远程互联网交互的方法 和模式, 但不能解决工程教育中对远程创新 实验的全面需求, 研究结果仅能为类似复杂 问题的解决提供方法借鉴而非完整方案。

\section{致谢}

本文为江苏省教育科学 “十二五” 规划 项目《应用型人才创新实验平台的国际合作 模式与实践》 $(D / 2013 / 01 / 130)$ 、江苏省2015 年研究生教育教学改革研究与实践课题《工 程类专业型硕士实践教学的网络交互模式 探索》以及江苏省政府留学奖学金（JS2013-326）的阶段性成果之一。

\section{References}

[1] Q. Wang, Designing a web-based constructivist learning environment, Interactive Learning Environments. vol. 17,pp.1-13, 2009.

[2] Y. Rui, P. Lili, Strategies of knowledge sharing in web 2.0 personal learning environment and their Evaluation, Open Education Research, vol. 21, pp.78-88, 2015.

[3] J.F. Guo, Z.Q. Ma, The design of interactive platform for the course of the
Internet of things based on Zigbee Technology, course education research, vol.7, pp.221-222, 2014.

[4] Y.H. Zhang, L. Wang, and L. L, Investigation and analysis of the network interaction between college students and teachers, Journal of Yangtze University (Social Science), vol.37, pp. 139- 141, 2014.

[5] G. Lurie, P. Sydelko, and T. Taxon, An object-oriented GIS toolkit for webbased and dynamic decision analysis applications, Journal of Geographic Information and Decision Analysis, vol. 6, pp. 108-116, 2002.

[6] M. Bertolotto, J.D. Carswell, L. Geown, e-spatial TM technology for spatial analysis and decision making in web-based land information management systems, Journal of Geographic Information and Decision Analysis, vol. 5, pp.95-114, 2001.

[7] P.J. Atkinson, D.J. Unwin, Density and local attribute estimation of an infectious disease using MapInfo, Computers \& Geosciences, vol.28, pp. 1095-1105,2002.

[8] Q.J. Zhu, J. Chen. MapXtreme resolution for internet publication of natural disaster information in earthquake engineering based on ANN, Systems Engineering Procedia, vol.1, pp.4-9, 2011.

[9] Q.J. Zhu, B. Hejmanowska, Analysis of GIS-based spatial variability and risk assessment, Journal of Chemical and Pharmaceutical Research, vol.5, pp.372380, 2013.

[10]A. Srivastava, B.N. Nagpal, R. Saxena, GIS based malaria information management system for urban malaria scheme in India, Computer Methods and Programs in Biomedicine, vol.71, pp.6375, 2003.

[11]H.L. Yu, L. Wu, Y. Liu, etal., A study of integration between GIS and GIS-based model based on web services, Journal of Chongqing Jianzhu University, vol. 28, pp.24-28, 2006. 